\title{
A systematic and prospectively validated approach for identifying synergistic drug combinations against malaria
}

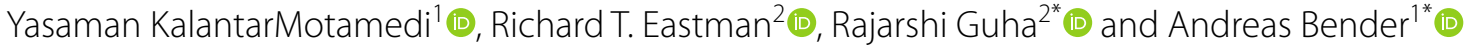

\begin{abstract}
Background: Nearly half of the world's population (3.2 billion people) were at risk of malaria in 2015, and resistance to current therapies is a major concern. While the standard of care includes drug combinations, there is a pressing need to identify new combinations that can bypass current resistance mechanisms. In the work presented here, a combined transcriptional drug repositioning/discovery and machine learning approach is proposed.

Methods: The integrated approach utilizes gene expression data from patient-derived samples, in combination with large-scale anti-malarial combination screening data, to predict synergistic compound combinations for three Plasmodium falciparum strains (3D7, DD2 and HB3). Both single compounds and combinations predicted to be active were prospectively tested in experiment.

Results: One of the predicted single agents, apicidin, was active with the AC50 values of $74.9,84.1$ and $74.9 \mathrm{nM}$ in 3D7, DD2 and HB3 P. falciparum strains while its maximal safe plasma concentration in human is $547.6 \pm 136.6 \mathrm{nM}$. Apicidin at the safe dose of $500 \mathrm{nM}$ kills on average $97 \%$ of the parasite. The synergy prediction algorithm exhibited overall precision and recall of 83.5 and $65.1 \%$ for mild-to-strong, 48.8 and $75.5 \%$ for moderate-to-strong and 12.0 and $62.7 \%$ for strong synergies. Some of the prospectively predicted combinations, such as tacrolimus-hydroxyzine and raloxifene-thioridazine, exhibited significant synergy across the three $P$. falciparum strains included in the study.
\end{abstract}

Conclusions: Systematic approaches can play an important role in accelerating discovering novel combinational therapies for malaria as it enables selecting novel synergistic compound pairs in a more informed and cost-effective manner.

Keywords: Synergy prediction, Malaria, Machine learning, Compound combination modelling, Transcriptional drug repositioning, Synergistic anti-malaria compound combinations

\section{Background}

While recently progress has been made towards the reduction of malaria related morbidity and mortality, an increasing at-risk population and insecticide resistant vectors hamper eradication efforts. In 2015, nearly half of the world's population ( 3.2 billion people) were at risk of contracting malaria, and 97 countries and territories

\footnotetext{
*Correspondence: guhar@mail.nih.gov; ab454@cam.ac.uk

${ }^{1}$ Centre for Molecular Informatics, Department of Chemistry, University

of Cambridge, Lensfield Road, Cambridge CB2 1EW, UK

2 Division of Preclinical Innovation, National Center for Advancing

Translational Sciences, National Institutes of Health, 9800 Medical Center

Drive, Rockville, MD 20852, USA
}

had ongoing malaria transmission [1]. Most malaria cases and deaths occur in sub-Saharan Africa [1]; however, Asia, Latin America, and, to a lesser extent, the Middle East and parts of Europe are also at risk. In particular, the emergence and spread of drug-resistant parasites continues to limit the effective lifespan of current anti-malarial drugs [2].

Drug resistance has reduced the effectiveness of previous standard therapies for malaria, such as chloroquine and sulfadoxine-pyrimethamine $[3,4]$. Today, artemisinin-based combination therapy (ACT) is frequently utilized, nearly universally in endemic regions, to reduce the selection of drug-resistant parasites [2]. Currently,

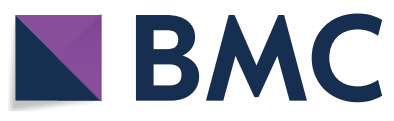

C The Author(s) 2018. This article is distributed under the terms of the Creative Commons Attribution 4.0 International License (http://creativecommons.org/licenses/by/4.0/), which permits unrestricted use, distribution, and reproduction in any medium, provided you give appropriate credit to the original author(s) and the source, provide a link to the Creative Commons license, and indicate if changes were made. The Creative Commons Public Domain Dedication waiver (http://creativecommons.org/ publicdomain/zero/1.0/) applies to the data made available in this article, unless otherwise stated. 
five artemisinin-based combinations (ACT) are available for the treatment of uncomplicated Plasmodium falciparum malaria, namely artemether and lumefantrine, artesunate and amodiaquine, artesunate and mefloquine, dihydroartemisinin and piperaquine, as well as artesunate and sulfadoxine-pyrimethamine [5]. New combinations (such as artesunate and pyronaridine) have also recently been registered for use in particular countries [5]. In order to avoid drug resistance, combination therapies comprised of single agents with different modes of actions are usually indicated. However, this is not always true since among the ACT medicines, some combinations such as mefloquine and lumefantrine act on similar pathways to those of artemisinin-derived drugs [6]. Recently, reduced response to ACT has been observed which indicates an urgent need for new combination therapies [7].

In this regard, a high-throughput combination screening study has recently been performed for malaria to provide experimental evidence for the efficacy of combinations [6]. However, due to the combinatorial explosion of the number of combinations possible, using a computational rationale for optimal selection of compound combinations can save cost and effort by providing a way to prioritize subsets of combinations from many possible compound pairs. Such compound combination modelling approaches have recently emerged for other diseases, particularly cancer $[8,9]$; however there have been relatively few studies focusing on malaria. The studies which have been published include in silico screening of targets and molecular docking to find novel anti-malarial agents [10-12], and a drug synergy visualisation tool for malaria [13]. The machine learning approaches applied in the malaria field are usually for diagnostic purposes [14], or have other aims such as improving docking scores [15] and identifying a malaria transmission model [16]. However, this excludes learning from large scale in vitro malaria screening data to predict novel anti-malarial combination therapies, which is the aim of this study. In this work, a novel systematic approach is proposed for identifying compounds that boost human response to severe malaria and combination of compounds that show synergy in the malaria in vitro system (Fig. 1). The

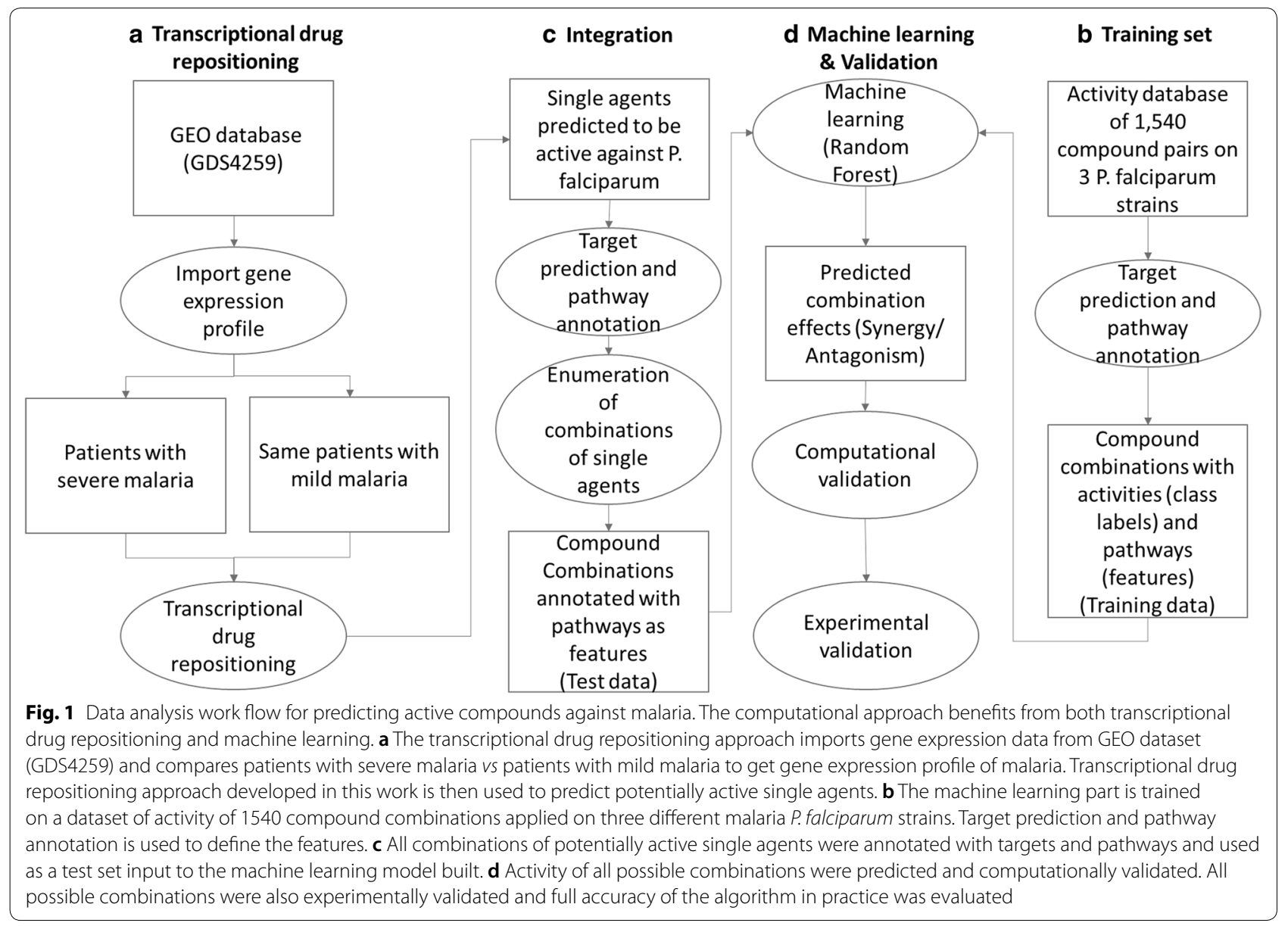


former was achieved via a transcriptional drug repositioning approach for shortlisting single agents and the later was achieved with a machine learning approach trained on in-vitro malaria screening data.

Transcriptomic data has been used previously for drug repositioning $[17,18]$, as well as for the selection of combination therapies in other areas [19]. There have been some transcriptional drug repositioning studies published for infectious diseases; $[20,21]$ however it has not been previously employed for malaria to the best knowledge of the authors. The usage of transcriptional data for compound selection is generally based on the hypothesis that compounds that affect differentially expressed genes of a disease in the opposite way to the disease itself ('anticorrelated gene signatures') have therapeutic potential for treating that particular disease. Experimental evidence supporting this approach has been presented in several studies, such as the repositioning of the antiulcer drug cimetidine for lung cancer [22], and of the antiepileptic topiramate for inflammatory bowel disease (IBS) [22]. In the present work, the human response to severe malaria has been derived in a different way compared to previous studies, namely gene expression data of blood samples of children with severe malaria was compared to gene expression signatures of the same children presented 1 month later, with mild malaria symptoms. Next, 201,776 compound gene signatures extracted from the recently published LINCS database [23] were compared to the malaria severity signature. This leads to a rank ordered list of compounds with anti-correlated gene signatures with malaria severity signature.

In this work, apart from the novelty of the application of transcriptional drug repositioning to malaria, previous approaches are also extended by utilising pathway annotations and in silico target predictions $[24,25]$ to the compound selection step. In this way, both biological readouts and on target bioactivity information are used to increase the signal and to have a more accessible understanding of compound action at hand. While understanding gene expression signatures can be rather complex, involving the up- and downregulation of pathways as well as the activity on individual proteins can often be more readily understood.

To this end, the in vitro combination screening data from 1540 compound pairs against three P. falciparum laboratory strains (3D7, DD, HB3) were utilized as a training set for the machine learning approach. To integrate the transcriptional drug repositioning, the machine learning and pathway annotation approach, synergistic combination pairs were selected from the single agents that were discovered in the preceding transcriptional drug repositioning approach. The activity predictions of individual compounds, and subsequently compound pairs, have been followed by prospective experimental validation, which resulted in the identification of novel single agent and synergistic compound combinations against $P$. falciparum. Hence, the results suggest that this method may be useful for prioritising new drug combinations for treating malaria.

\section{Methods}

Data

Training data previously generated at NCATS [6] included 1540 combinations of 56 individual compounds measured on three different $P$. falciparum strains, namely 3D7, DD2 and HB3 (https://tripod.nih.gov/matrix-clien $\mathrm{t} / \mathrm{p}=183$, with assay IDs: $1463,1464,1465)$. For each of the combinations, the $\gamma$ measure was calculated to characterize synergy between the pairs as described previously [26]. Values for $\gamma<1$ represent synergy, $\gamma=1$ represents additivity and $\gamma>1$ represents antagonism. Combination screening quality is characterized by a percombination QC metric, whose value ranges from 1 to 18 , where 1 represents the highest quality data and 18 represents lowest data quality. Only data points with QC values less than 3 were used for subsequent analyses.

\section{Transcriptional drug repositioning approach}

In order to select new compounds for screening, first a combined bioinformatics and cheminformatics approach developed earlier [27] was used to predict single compounds with activity against malaria. For this purpose GEO dataset [28] GDS4259 was downloaded using the GEOImporter [29] tool of GenePattern [30]. This dataset contains data derived from peripheral blood samples from five Malawian children with severe $P$. falciparum malaria, compared to the same children who presented with a mild case of malaria 1 month later [31]. This comparison gives rise to the gene signature of severe malaria, and it was used in this work as a disease signature. The controls were chosen as human host cells that have responded in a curative manner to the disease, and hence decreased disease severity. This choice was made to make sure that the host response is present in the malaria severity signature. In the next steps, it was searched for drugs to reverse this severity signature which is expected to change a state of severe malaria to convalescent and help boost the host response.

In order to find compounds that have strong negative connectivity (anti-correlation) with the gene signature of malaria severity, compound-gene expression profiles from the LINCS database (Phase I, GEO dataset GSE92742) [23] were utilized. This calculation was performed based on a modified Gene Set Enrichment Analysis (GSEA) [32]. Considering the top 50 and bottom 50 up- and down-regulated genes of every compound, the 
calculation derives a score describing the strength of connection of compound signatures with a given disease profile. The scoring system leads to a rank ordered list of compounds that are assumed to be able to reverse the gene expression signature present in the malaria severity signature. Fifty highly scored compounds were shortlisted as single agent candidates.

\section{Machine learning approach}

The machine learning part of the algorithm involved in-silico target prediction, pathway annotation, synergy model generation and computational validation as follows.

\section{Target prediction}

Firstly, protein targets of all the 56 compounds in the training dataset were predicted based on an updated version of target prediction algorithm developed previously [24]. The algorithm was retrained on compound/target pairs from ChEMBL [33] v17 and compound targets were predicted using a Laplacian Modified Naïve Bayes scoring system $[25,32]$. Next, protein targets were predicted for the 50 shortlisted compounds from the transcriptional drug repositioning approach. The predicted protein targets with a score over 14 were selected as potential protein targets of each compound. The score cut-off gave rise on average to six targets for a compound, which is a reasonable number of targets for a ligand, based on previous analyses [34].

\section{Pathway annotation}

Next, pathways enriched by the targets of each compound were identified. For this purpose, 2010 human pathways and their associated genes from the Biosystems [35] database were extracted. The equivalent Entrez gene IDs of each protein target of compounds were retrieved using Biomart [36]. For each compound a list of, on average, six gene IDs that the compound is predicted to interact with (the 'compound gene signature') was obtained. Then, the number of shared genes between each compound gene signature and gene members of each of 2010 pathways were found and stored as a raw integer score in a sparse vector, resulting in a vector with 2010 values for each compound, termed the 'pathway signature of a compound'.

Next, for each combination of compound 'a' and compound ' $b$ ' in the combination dataset, a descriptor vector was created by merging their compound pathway signatures using the following equation:

$$
p_{i, a, b}=\left(P_{i, a}+1\right) *\left(P_{i, b}+1\right)
$$

where $P_{i, a}$ and $P_{i, b}$ are the $i$ th value in compound 'a' or 'b' pathway signature which is the number of shared genes between $i$ th pathway and compound 'a' or ' $\mathrm{b}$ ' gene signature. $p_{i, a, b}$ is the $i$ th value in the pathway signature of combination of compound 'a' and compound 'b'. Each term was added with one to avoid $p_{i, a, b}=0$ in cases where one of $P_{i, a}$ or $P_{i, b}$ equals zero. In this way, it is avoided to filter out the effect of one compound on the pathway when the other one has no effect on that pathway. In other words, the above formula gives a high score to pathways that are shared between both compounds which is the product of the score of both compounds, while also not neglecting the pathways that are only hit by one compound.

\section{Synergy model generation}

Using the above formula, a training file was created for each pair of compound pathway signatures labelled with synergy or no synergy, depending on gamma and QC. Pairs of compounds with gamma value $<0.975$ and $\mathrm{QC} \leq 3$ were marked as synergistic and gamma $>1.025$ and $\mathrm{QC} \leq 3$ were marked as antagonistic. The 0.975 and 1.025 cut-offs for gamma were chosen to avoid the additivity window. All the data with $\mathrm{QC}>3$ was discarded. Next, the training file was used as input for a Random Forest [37] algorithm consisting of 200 trees as implemented in Matlab (2015B) using the TreeBagger [38] function. This algorithm was hence able to predict the synergy of a compound combination, based on the pathways that are hit by this given pair of compounds.

\section{Computational validation for drug combination}

As a computational step to validate the approach prior experiments, cross validation was used to evaluate performance of the methods. The number of true positives (TP), false positives (FP) and false negatives (FN) were used to calculate precision $\left(\frac{\mathrm{TP}}{\mathrm{TP}+\mathrm{FP}}\right)$ and recall $\left(\frac{\mathrm{TP}}{\mathrm{TP}+\mathrm{FN}}\right)$. The tenfold cross validation was applied on the original data which was yielding $66 \%$ precision, $61 \%$ recall and $61 \%$ for the F-measure over the two classes of synergy and antagonism, averaged over all three strains.

\section{Prospective utilisation of the synergy model}

Next, combinations of compounds in the shortlist produced using the above transcriptional drug repositioning approach were annotated with pathways using Eq. 1 and fed to the random forest model as a prospective test set (in order to predict likely synergistic compound combinations for this new set of compounds). The random forest model hence predicted synergies and antagonism for compound combinations, and predicted pairs were ranked by their probability of being synergistic. The top 17 compound combinations, representing 14 unique single agents, were selected for experimental validation as summarized in Additional file 1: Table S2. 


\section{Experimental validation of compounds for malaria}

The $P$. falciparum parasite lines were maintained in in vitro culture conditions as described previously [39]. Methods for the SYBR qHTS and calculation of $\mathrm{IC}_{50}$ and definition of curve classes have been used as in previous work [39-41], as was the plating of compounds for combination screening using acoustic dispense methods [42]. As shown in the Additional file 2: Figure S1, classes of activity were assigned based on growth inhibition curves where -1.1 shows a complete curve as well as high efficacy, -1.2 represents a complete curve but only partial efficacy, -2.1 symbolizes a partial curve which however exhibits high efficacy, -2.2 represents a partial curve with only partial efficacy and -2.3 represents a partial curve with high efficacy, but only poor curve fit. All HTS assays were read at $72 \mathrm{~h}$. Percent response values shown in matrix heat maps represent relative growth as obtained from SYBR Green fluorescence intensity values normalized to controls. Synergy metrics for combinations were computed as described [42]. Data generated as part of the prospective validation can be accessed at https ://tripod.nih.gov/matrix-client/?p=1261.

\section{Results}

\section{Prospective validation of single agents}

With the aid of the transcriptional drug repositioning approach, 14 single agents (structures are displayed in Fig. 2) were prioritized and subsequently tested experimentally for their activity against the 3D7, DD2 and HB3 strains of $P$. falciparum. The results of the screen are summarised in Table 1, where chromomycin-a3 (CHR), fulvestrant (FUL), apicidin (API), ingenol (ING) and tacrolimus (TAC) exhibited full inhibition in the dose response curves in low concentrations. Of those, chromomycin-a3, fulvestrant and apicidin were active in nanomolar doses in all the three strains tested, with the average AC50 values of 11, 67 and $78 \mathrm{nM}$. JX-401 (JX4), raloxifene (RAL), hydroxyzine (HYD), thioridazine (THI), KIN001-244 (KIN), PI 828 (PI8), megestrol (MEG) exhibited complete or partial activity in different individual strains. Monorden (MON) and chelidonine (CHE) exhibited partial or single dose activity across all strains and were not progressed further. All experimental results data can be accessed at https://tripod.nih.gov/matri $\mathrm{x}$-client/?p=1261.

\section{Prospective validation of drug combinations}

Thirty five compound pairs were selected based on synergy prediction model as well as single agent screening data and were experimentally tested against $P$. falciparum strains of 3D7, DD2 and Hb3. Table 2 represents predicted probability for synergy of each of combinations in each strain (calculated before experiments) as well as synergy metric, $\gamma$ [26] values, (calculated after experiments). Among 35 combination pairs that have been tested in this study, 28 were predicted to be synergistic at least in one strain. Among those 28 predicted pairs, 27 were showing mild-strong synergy, 17 were showing moderate-strong synergy and 8 were showing strong synergy at least in one strain. As one can see in the Table 3, only one of five currently used ACT medicines (mefloquine-artesunate) shows strong synergy on all the three strains and one (amodiaquine-artesunate) has strong synergy only on the HB3 strain. Precisions and recall measures were utilized to measure the accuracy of synergy predictions compared to experiments, the results of which are shown in Table 4. For this purpose various gamma cut-offs were used to signify mild-to-strong (gamma $\leq 0.995)$, moderate-to-strong $($ gamma $\leq 0.975)$ and strong (gamma $\leq 0.95)$ synergies. The overall average precision and recall of experiments over the three strains were 83.5 and $65.1 \%$ for mild-to-strong, 48.8 and $75.5 \%$ for moderate-strong and 12.0 and $62.7 \%$ for strong synergies.

A network representation of compound pairs were provided in Fig. 3 to represent synergy strength of different compound pairs and identify compounds that have highest number of synergies with other compounds. It can be seen that tacrolimus-hydroxyzine and raloxifene-thioridazine, represent the most synergistic compound pairs followed by ingenol-tacrolimus, tacrolimus-fulvestrant, tacrolimus-apicidin and raloxifene-megestrol pairs. Tacrolimus is synergistic with highest number of compounds (three).

The experimental validation of the most synergistic compound combinations in the $P$. falciparum strains were depicted in Fig. 4 using the HSA synergy model [43] to identify the optimal synergy doses. In the DD2 assay, the most synergistic combination was tacrolimus with hydroxyzine, where $1.250 \mu \mathrm{M}$ hydroxyzine and $0.156 \mu \mathrm{M}$ tacrolimus demonstrated highest synergy of -0.45 in the HSA system (Fig. 4b). In the 3D7 and HB3 strains on the other hand, raloxifene together with thioridazine was the most synergistic compound pair (Fig. 4d). Here for the 3D7 strain $1.250 \mu \mathrm{M}$ raloxifene and $2.5 \mu \mathrm{M}$ thioridazine achieved maximum synergy of -0.85 , while in the HB3 assay this is the case for concentrations of $2.5 \mu \mathrm{M}$ of raloxifene and $10 \mu \mathrm{M}$ of thioridazine with synergy of -0.94 . However, high synergy values were also observed at other compound concentrations, such as $2.5 \mu \mathrm{M}$ of raloxifene with $5 \mu \mathrm{M}$ of thioridazine, as well as $1.25 \mu \mathrm{M}$ of raloxifene and $10 \mu \mathrm{M}$ thioridazine with synergy values of -0.78 and -0.72 , respectively. 
<smiles>Nc1ccc(-c2cccc3c(=O)cc(N4CCOCC4)oc23)cc1</smiles>

PI 828 (PI8)

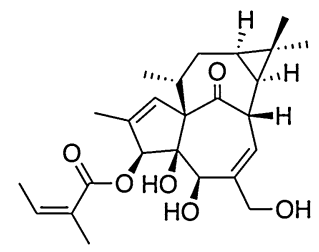

Ingenol (ING)

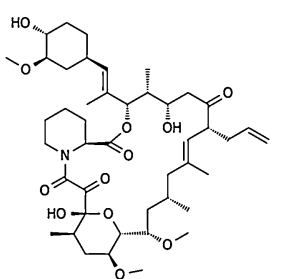

Tacrolimus (TAC)<smiles>CO[C@@H](C)C[C@H]1O[C@H]1/C=C\C=C/C(=O)Cc1c(Cl)c(O)cc(O)c1C(=O)O</smiles>

Monorden(MON)

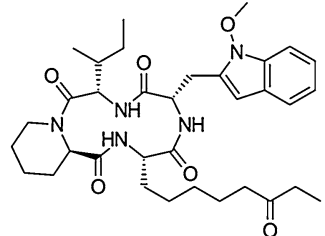

Apicidin (API)<smiles>O=C(c1ccc(OCCN2CCCCC2)cc1)c1c(-c2ccc(O)cc2)sc2cc(O)ccc12</smiles>

Raloxifene (RAL)<smiles>CN1Cc2c(ccc3c2OCO3)[C@@H]2[C@@H](O)Cc3cc4c(cc3[C@]21C)OCO4</smiles>

Chelidonine (CHE)<smiles>OCCOCCN1CCN(C(c2ccccc2)c2ccc(Cl)cc2)CC1</smiles>

Hydroxyzine (HYD)<smiles>CSc1ccc2c(c1)N(CCC1CCCCN1C)c1ccccc1S2</smiles>

Thioridazine(THI)
JX-401 (JX4)<smiles>COc1cc(SC)ccc1C(=O)N1CCC(Cc2ccccc2)CC1</smiles><smiles>CC(=O)[C@]1(O)CC[C@H]2[C@@H]3C=C(C)C4=CC(=O)CC[C@]4(C)[C@H]3CC[C@]21C</smiles>

Megestrol (MEG)

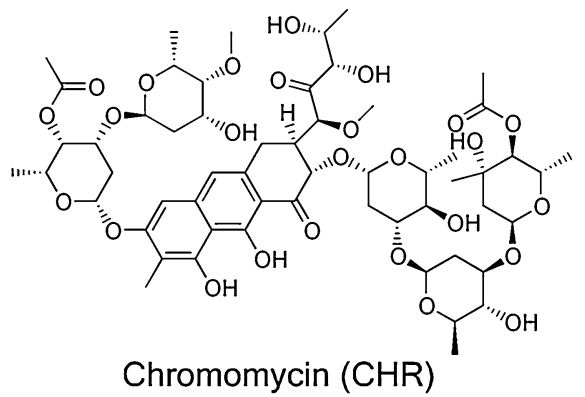<smiles>CC12CCC3c4ccc(O)cc4C[C@H](CCCCCCCCCS(=O)CCCC(F)(F)C(F)(F)F)[C@H]3[C@@H]1CCC2O</smiles>

Fulvestrant (FUL)

Fig. 2 Chemical structures of predicted single agents against malaria. All the structures were experimentally tested in the P. falciparum screen (see Table 1 for results)

\section{Discussion}

\section{Mechanistic justification of the compounds}

In this section, differentially expressed genes and pathways that are involved in the malaria severity signature as well as potential modes of action on the target and pathway level are discussed. It is important to consider that human targets and human pathways are used as the gene expression data utilized is also from human sources. This will enable studying the effect of the compounds on the host.

In the malaria severity signature, several immune response related genes were down-regulated. In other words, these genes were up-regulated in patients that responded to the disease in a curative way and hence recovered from the disease. The computational transcriptional drug repositioning approach is looking for 
Table 1 Experimental validation of predicted anti-malarial single agent compounds

\begin{tabular}{|c|c|c|c|c|c|c|c|}
\hline Compound & $\begin{array}{l}\text { Class } \\
\text { 3D7 }\end{array}$ & $\begin{array}{l}\text { Class } \\
\text { DD2 }\end{array}$ & $\begin{array}{l}\text { Class } \\
\text { HB3 }\end{array}$ & $\begin{array}{l}\text { AC50 } \\
3 \mathrm{D} 7\end{array}$ & $\begin{array}{l}\text { AC50 } \\
\text { DD2 }\end{array}$ & $\begin{array}{l}\text { AC50 } \\
\text { HB3 }\end{array}$ & Average AC50 \\
\hline $\mathrm{CHR}$ & -1.1 & -1.1 & -1.1 & 0.0119 & 0.0106 & 0.0106 & 0.011 \\
\hline FUL & -1.1 & -1.1 & -1.1 & 0.0335 & 0.0335 & 0.1332 & 0.0667 \\
\hline API & -1.1 & -1.1 & -1.1 & 0.0749 & 0.0841 & 0.0749 & 0.078 \\
\hline ING & -1.1 & -1.1 & -1.1 & 1.3324 & 1.495 & 1.495 & 1.4408 \\
\hline MON & -2.1 & -2.1 & -2.3 & 1.4956 & 1.0588 & 1.8828 & 1.4791 \\
\hline $\mathrm{RAL}$ & -1.1 & -1.1 & -2.1 & 0.7493 & 0.3347 & 4.7277 & 1.9372 \\
\hline TAC & -1.1 & -1.1 & -1.1 & 1.8821 & 3.7553 & 1.6774 & 2.4383 \\
\hline $\mathrm{CHE}$ & 1.3 & -2.3 & 1.3 & 0.0266 & 10.5839 & 0.0011 & 3.5372 \\
\hline$J X 4$ & -1.1 & -1.1 & -2.1 & 0.7493 & 0.0595 & 10.5839 & 3.7976 \\
\hline HYD & -1.1 & -1.1 & -2.1 & 2.6586 & 1.6774 & 11.8754 & 5.4038 \\
\hline $\mathrm{THI}$ & -2.1 & -2.1 & -2.1 & 10.5839 & 14.9502 & 14.9502 & 13.4948 \\
\hline MEG & -2.1 & -1.1 & -2.1 & 14.9502 & 1.8821 & 23.6945 & 13.5089 \\
\hline PI8 & -2.3 & -2.1 & -2.1 & 13.3244 & 11.8754 & 16.7744 & 13.9914 \\
\hline $\mathrm{KIN}$ & -2.1 & -2.1 & -2.1 & 13.3244 & 21.1177 & 16.7744 & 17.0722 \\
\hline
\end{tabular}

The fitted AC50 values $(\mu \mathrm{M})$ for the single drugs as well as classes of activity are listed. AC50 indicates $50 \%$ maximal response (for inhibition or agonism) and is calculated by fitting a 4-parameter logistic model to the concentration, response data. Curve classes are defined in Additional file 2: Figure S1

Classes that are identified as -1.1 are highlighted in italics as it signifies complete curve as well as high efficacy

compounds that reverse the malaria severity signature and up-regulate the immune response genes to help boost the immune response. The immunity related genes that are down-regulated in the malaria severity signature are: PRF1, GNLY, OAS2, MX1, OAS3 and CCL5. PRF1 encodes a protein with structural similarities to complement component C9 that is important in immunity [44]. GNLY protein is present in cytotoxic granules of cytotoxic $\mathrm{T}$ lymphocytes and natural killer cells, and it has antimicrobial activity against $M$. tuberculosis and other organisms [44]. OAS2 and OAS3 encode a members of the 2-5A synthetase family, essential proteins involved in the innate immune response to viral infection. These molecules activate latent RNase $\mathrm{L}$, which results in viral RNA degradation and the inhibition of viral replication. They play significant role in the inhibition of cellular protein synthesis and viral infection resistance [46]. MX1 participates in the cellular antiviral response. CCL5 is one of several chemokine genes. Chemokines form a superfamily of secreted proteins involved in immunoregulatory and inflammatory processes. SLPI is also up-regulated in the malaria severity signature. Its inhibitory effect contributes to the immune response [44]. Pathways associated with mild malaria include the type I interferon-mediated signalling pathway, $\mathrm{T}$ cell activation and other pathways representing many aspects of immune activation [31]. The malaria severity signature contains six genes that were associated with severe malaria, including carbonic anhydrase 1 (CA1), G-protein-coupled receptor 89B (GPR89B), lipocalin 2 (LCN2), thymidine kinase 1 (TK1), small nucleolar RNA, C/D box 30 (SNORD30), and TBC1 domain family member 3 (TBC1D3) $(\mathrm{P} \leq 0.05$; fold change of $\geq 1.5)$ [31]. Thymidine kinase 1 was recently found to be a biomarker of cerebral malaria susceptibility in the murine model [45], and carbonic anhydrase, reflects the blood's abnormal acid base environment during severe disease. Further discussion of genes and pathways in the malaria severity signature has been published before [31].

Among the most potent single agents, tacrolimus has targets with established links to malaria. Tacrolimus has been previously studied for its potential anti-malarial activity by binding to PfFKBP35 and PvFKBP35 proteins of the parasite $[46,47]$; however, the synergistic combination with hydroxyzine has not been reported before. Tacrolimus as well as apicidin were predicted to target “TGF-beta receptor signalling" pathway of human host. It is known that tacrolimus enhances TGF-Beta expression [48] while production of this protein is inversely correlated with severity of murine malaria infection [49]. Activation of another member of this pathway namely latent TGF-beta is suggested as a novel mechanism for direct modulation of the host response by malaria parasites [50]. Hence, modulation of TGF-Beta signalling may be a novel mechanism of tacrolimus that directly affects the host. Moreover, in vivo validation of apicidin against Plasmodium berghei in mice is evident from the literature [51].

On the other hand, pathways enriched by the predicted targets of the selected single agents according to 
Table 2 Synergy prediction and synergy scores based on experiments

\begin{tabular}{|c|c|c|c|c|c|c|c|}
\hline Compund 1 & Compound 2 & $\begin{array}{l}\text { 3D7 } \\
\text { Pred }\end{array}$ & $\begin{array}{l}\text { 3D7 } \\
\text { Gamma }\end{array}$ & $\begin{array}{l}\text { DD2 } \\
\text { Pred }\end{array}$ & $\begin{array}{l}\text { DD2 } \\
\text { Gamma }\end{array}$ & $\begin{array}{l}\text { HB3 } \\
\text { Pred }\end{array}$ & $\begin{array}{l}\text { HB3 } \\
\text { Gamma }\end{array}$ \\
\hline TAC & HYD & 0.309 & $0.948^{* *}$ & 0.189 & $0.885^{* *}$ & 0.501 & $0.951^{*}$ \\
\hline FUL & TAC & 0.627 & $0.961^{*}$ & 0.612 & $0.908^{* *}$ & 0.659 & 0.989 \\
\hline ING & TAC & 0.820 & $0.954^{*}$ & 0.674 & $0.917^{* *}$ & 0.854 & $0.954^{*}$ \\
\hline FUL & MEG & 0.722 & 1.007 & 0.583 & $0.918^{* *}$ & 0.745 & $0.970^{*}$ \\
\hline MEG & HYD & 0.784 & 0.985 & 0.629 & $0.920^{* *}$ & 0.738 & 0.988 \\
\hline KIN & HYD & 0.475 & 0.984 & 0.371 & $0.938^{* *}$ & 0.477 & 0.977 \\
\hline API & TAC & 0.416 & $0.975^{*}$ & 0.685 & $0.942^{* *}$ & 0.523 & $0.946^{* *}$ \\
\hline FUL & RAL & 0.792 & 0.980 & 0.739 & $0.952^{*}$ & 0.825 & $0.966^{*}$ \\
\hline API & HYD & 0.806 & 0.987 & 0.717 & $0.952^{*}$ & 0.501 & $0.968^{*}$ \\
\hline FUL & $\mathrm{THI}$ & 0.651 & 0.982 & 0.714 & $0.954^{*}$ & 0.540 & $0.960^{*}$ \\
\hline $\mathrm{THI}$ & RAL & 0.549 & $0.931^{* *}$ & 0.698 & $0.956^{*}$ & 0.375 & $0.911^{* *}$ \\
\hline $\mathrm{Pl} 8$ & HYD & 0.278 & 0.977 & 0.293 & $0.961^{*}$ & 0.171 & 0.993 \\
\hline FUL & HYD & 0.788 & $0.965^{*}$ & 0.747 & $0.962^{*}$ & 0.734 & $0.951^{*}$ \\
\hline TAC & RAL & 0.483 & 0.995 & 0.342 & $0.964^{*}$ & 0.612 & $0.962^{*}$ \\
\hline ING & $J X 4$ & 0.859 & 0.980 & 0.740 & $0.968^{*}$ & 0.826 & $0.975^{*}$ \\
\hline MEG & RAL & 0.795 & $0.937^{* *}$ & 0.648 & $0.969^{*}$ & 0.791 & $0.966^{*}$ \\
\hline ING & RAL & 0.836 & $0.964^{*}$ & 0.658 & $0.975^{*}$ & 0.793 & $0.966^{*}$ \\
\hline API & RAL & 0.814 & 0.997 & 0.697 & 0.976 & 0.472 & 0.992 \\
\hline $\mathrm{CHR}$ & HYD & 0.633 & 1.001 & 0.238 & 0.979 & 0.644 & 0.991 \\
\hline KIN & $J X 4$ & 0.510 & 0.987 & 0.530 & 0.982 & 0.479 & 0.989 \\
\hline API & JX4 & 0.761 & 1.020 & 0.723 & 0.984 & 0.495 & $0.970^{*}$ \\
\hline $\mathrm{CHR}$ & TAC & 0.418 & 0.99 & 0.163 & 0.987 & 0.445 & 0.996 \\
\hline JX4 & HYD & 0.498 & 0.984 & 0.503 & 0.987 & 0.498 & 0.986 \\
\hline $\mathrm{Pl} 8$ & ING & 0.551 & $0.975^{*}$ & 0.443 & 0.991 & 0.499 & 0.989 \\
\hline $\mathrm{Pl} 8$ & $J X 4$ & 0.238 & 0.990 & 0.621 & 0.994 & 0.214 & 0.981 \\
\hline $\mathrm{Pl} 8$ & RAL & 0.369 & 1.014 & 0.293 & 0.997 & 0.126 & 0.995 \\
\hline $\mathrm{CHR}$ & $J X 4$ & 0.709 & 0.990 & 0.355 & 0.999 & 0.608 & 0.991 \\
\hline API & MEG & 0.776 & 0.981 & 0.632 & 1.004 & 0.582 & 0.979 \\
\hline $\mathrm{CHR}$ & MEG & 0.817 & 0.995 & 0.568 & 1.010 & 0.791 & 0.985 \\
\hline $\mathrm{Pl} 8$ & TAC & 0.283 & 0.986 & 0.205 & 1.014 & 0.118 & 0.983 \\
\hline API & $\mathrm{CHR}$ & 0.785 & 0.999 & 0.722 & 1.014 & 0.525 & 0.998 \\
\hline $\mathrm{CHR}$ & RAL & 0.785 & 0.990 & 0.442 & 1.019 & 0.695 & 1.004 \\
\hline $\mathrm{Pl} 8$ & MEG & 0.491 & $0.972^{*}$ & 0.366 & 1.021 & 0.272 & $0.966^{*}$ \\
\hline ING & HYD & 0.869 & 0.985 & 0.699 & 1.021 & 0.810 & 0.992 \\
\hline Pl8 & KIN & 0.246 & 0.989 & 0.348 & 1.046 & 0.197 & 0.997 \\
\hline
\end{tabular}

This table represents gamma $(\gamma)$ values as a metric for calculation of synergy as well as predicted probability of synergy for three different $P$. falciparum strains. Values of $\gamma \leq 0.95$ represent strong synergy and were marked with ${ }^{* *}$ and italic. Values of $0.95<$ http $<0.975$ were identified as moderate synergy and marked with * and italic, $0.975<\gamma<0.995$ were identified as mild synergy and marked with italic font. Prediction scores above 0.5 were true predictions and were marked with italic font

Biosystems pathways are depicted in Fig. 5 (also represented in Additional file 3: Figure S2 and listed in more detail in Additional file 4: Table S1). Figure 5 suggests that highest synergistic combinations such as tacrolimus with hydroxyzine (average $(\gamma)=0.928$ ), as well as raloxifene and thioridazine (average $(\gamma)=0.932$ ), target independent pathways rather than shared pathways. On the other hand, pairs of compounds that have the most similar pathway signature did not have strong synergy. As an example, tacrolimus-chromomycin, hydroxyzineKIN001244 and fulvestrant-megestrol are pairs that are clustered together (Fig. 5) but all of them are showing week synergies with average $\gamma$ values of $0.991,0.965$, and 0.966 . The data suggests that targeting independent pathways by compound pairs is privileged to targeting the same pathway multiple times for synergy to emerge. 
Table 3 Gamma synergy values for ACT as current synergistic therapies

\begin{tabular}{lllll}
\hline Compound 1 & Compound 2 & $\begin{array}{l}\text { 3D7 } \\
\text { Gamma }\end{array}$ & $\begin{array}{l}\text { DD2 } \\
\text { Gamma }\end{array}$ & $\begin{array}{l}\text { HB3 } \\
\text { Gamma }\end{array}$ \\
\hline Mefloquine & Artesunate & $0.911^{* *}$ & $0.786^{* *}$ & $0.797^{* *}$ \\
Amodiaquine & Artesunate & 0.993 & 1.064 & $0.955^{*}$ \\
Sulfadoxine & Artesunate & 0.986 & 1.016 & $0.936^{* *}$ \\
Lumefantrine & Artemether & 2.221 & 1.058 & 0.997 \\
Piperaquine & $\begin{array}{c}\text { Dihydroartemisinin } \\
\text { (DHA) }\end{array}$ & 0.999 & 1.007 & 0.999 \\
& & & & \\
\hline
\end{tabular}

This table shows synergy metric gamma for current anti-malarial combination therapies for comparison reason to the results provided in Table 2 (similar markings)

\section{Limitations of the study}

The limitation of transcriptional drug repositioning approaches that are gaining popularity is that they cannot be applied for predicting compounds that act on parasite directly. Some recent other applications of transcriptional drug repositioning approaches for finding antivirals took into account the effect of compounds on host cells $[20,21]$. This limitation is due to CMap and LINCS databases that are the gateways for transcriptional drug repositioning approaches. The LINCS database used in this work contains 201,776 compound treatment gene signatures that are applied on "human" cell lines and hence provides genes up/down-regulated in human tissue. Hence, it is not applicable to the parasites directly. Another limitation of the transcriptional drug repositioning approaches is that they are limited to the compounds that are included in LINCS or CMap database.

The use of transcriptional drug repositioning in the context of this project can facilitate identifying single agent compounds that help boost immunity of host to the parasite. Given that the combination of those compounds are expected to show synergy in the in vitro system as well, a two round selection process was performed. In the first round, single compounds were predicted to be active in blood cells of patients infected with severe malaria. In the second round, combinations of those single compounds were selected that were predicted to be synergistic on the parasite infected in vitro system containing host red blood cells. The final compound combinations were as a result of using both filtering methods. It needs to be considered that the in vitro system includes human red-blood cells and this means human pathways are present in the in vitro system. Moreover, the training data for synergies was generated based on the same in vitro platform of the final experimental tests which has human red blood cells infected with malaria parasite. Hence, the final combination sets are correctly predicted for the right platform.

One of the limitations of the study is the number of patient samples used in this work. Even though the gene expression samples of only five patients were used in the study, they were hand-picked among 119 patients participating in Blantyre malaria research. These patients were selected as they manifested clinical features of severe malaria but were dramatically recovered, having no clinical evidence of severe disease. They were also reported to be clear of peripheral blood parasites and bacterial meningitis at admission and after $48 \mathrm{~h}$ [31]. The authors that published the database claimed that "five severe/mild paired samples had sufficiently high-quality RNA for microarray and further analysis". However, the sample size was mentioned as a limitation of the study.

Generally, the application of computational biology approach introduced in this study is limited to early stage drug discovery/repurposing of single agents as well as combination therapies. Any of the findings of such early stage studies should be followed up with

Table 4 Precision and recall calculated for synergistic compounds

\begin{tabular}{|c|c|c|c|c|c|c|c|c|c|c|c|c|}
\hline \multirow[t]{2}{*}{ Strain } & \multicolumn{4}{|c|}{$\begin{array}{l}\text { Mild-to-strong synergy } \\
\text { (gamma } \leq 0.995)\end{array}$} & \multicolumn{4}{|c|}{$\begin{array}{l}\text { Moderate-to-strong synergy } \\
\text { (gamma } \leq 0.975 \text { ) }\end{array}$} & \multicolumn{4}{|c|}{ Strong synergy (gamma $\leq 0.95)$} \\
\hline & 3D7 & DD2 & HB3 & AVG & 3D7 & DD2 & HB3 & AVG & 3D7 & DD2 & HB3 & AVG \\
\hline True positives & 18 & 18 & 19 & 18.3 & 7 & 13 & 12 & 10.7 & 2 & 5 & 1 & 2.7 \\
\hline False negatives & 11 & 7 & 12 & 10.0 & 3 & 4 & 3 & 3.3 & 1 & 2 & 1 & 1.3 \\
\hline False positives & 5 & 4 & 2 & 3.7 & 16 & 9 & 9 & 11.3 & 21 & 17 & 20 & 19.3 \\
\hline Precision (\%) & 78.3 & 81.8 & 90.5 & 83.3 & 30.4 & 59.1 & 57.1 & 48.5 & 8.7 & 22.7 & 4.8 & 12.1 \\
\hline Recall (\%) & 62.1 & 72.0 & 61.3 & 64.7 & 70.0 & 76.5 & 80.0 & 76.2 & 66.7 & 71.4 & 50.0 & 66.7 \\
\hline F measure (\%) & 69.2 & 76.6 & 73.1 & 72.8 & 42.4 & 66.7 & 66.7 & 59.3 & 15.4 & 34.5 & 8.7 & 20.5 \\
\hline Training set synergies (\%) & 42.8 & 41.2 & 40.0 & 41.3 & 25.7 & 25.3 & 23.0 & 24.7 & 15.8 & 15.6 & 13.4 & 14.9 \\
\hline
\end{tabular}

Precision and recall was calculated for each of the three $P$. falciparum strains with respect to three different cut-offs for gamma to distinguish the performance of the algorithm for mild-to-strong, moderate-to-strong and strong synergy. As you can see in all of the predictions recall has been kept high between 50 and $80 \%$ depending the expected synergy level and the strain. The highest precision is for Mild-to-strong synergies ranging from 61.3 to $72 \%$. However, as the synergy level expectation is increased to Moderate-to-strong or only Strong synergies the Precision drops to $30-59 \%$ and $4.8-22.7 \%$ 


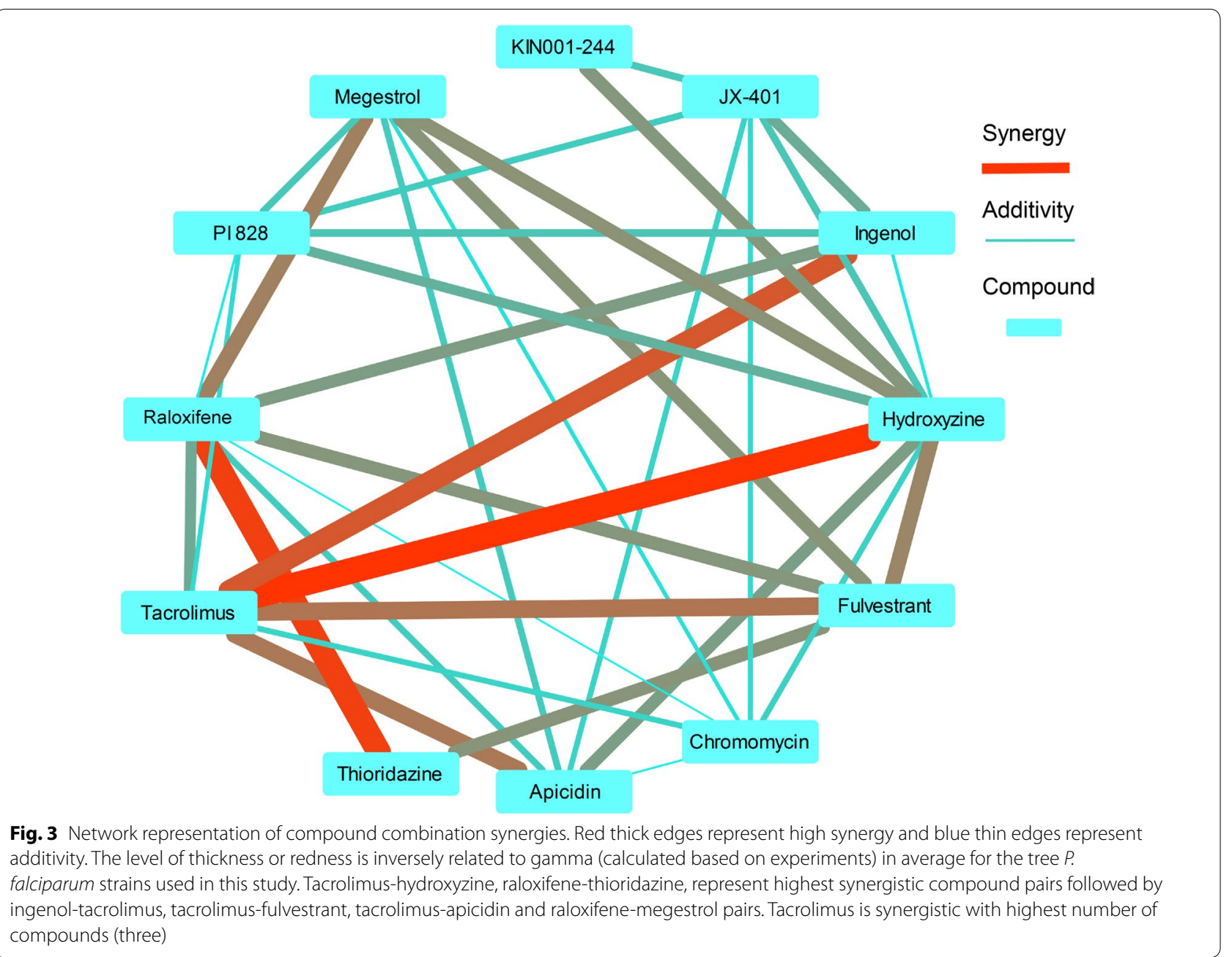

further lab and possibly animal studies to be able to be translated into clinical trials. However, the approach can be used on other diseases as well given availability of appropriate data.

\section{Performance evaluation and effect of gamma choice}

The choice of gamma affects sample size of the synergy class and hence it is a trade-off between the sample size and quality of samples (degree of synergy). Gamma cutoff of 0.975 marked in average between the three strains 379.3 out of 1540 combinations in the training set as synergistic while gamma cut-off of 0.95 reduced average number of synergistic instances to 229.7. Given the dimensionality of the data with 2010 features, higher gamma cut-off of 0.975 was chosen to keep the sample size in the synergy class as high as possible while avoiding the additivity window. To show that the gamma cut-off is relevant in this study, Table 3 shows gamma values of current combinational therapies. Only one of five currently used ACT medicines (mefloquine-artesunate) on all the three strains and one (amodiaquine-artesunate) on the HB3 strain have gamma values bellow 0.95 . Hence,

(See figure on next page.)

Fig. 4 Synergistic compound combinations identified against $P$. falciparum. Representation of dose-response matrix and HSA synergy model for tacrolimus-hydroxyzine $(\mathbf{a}, \mathbf{b})$ and raloxifene-thioridazine ( $\mathbf{c}, \mathbf{d})$ in the three parasite lines (3D7, DD2, HB3). The effect of increasing drug concentration from right to left and bottom to top was assessed in a $10 \times 10$ block size matrix $(\mathbf{a}, \mathbf{c})$. HSA synergy model represents in which concentrations maximum synergy occurs (b, d) 


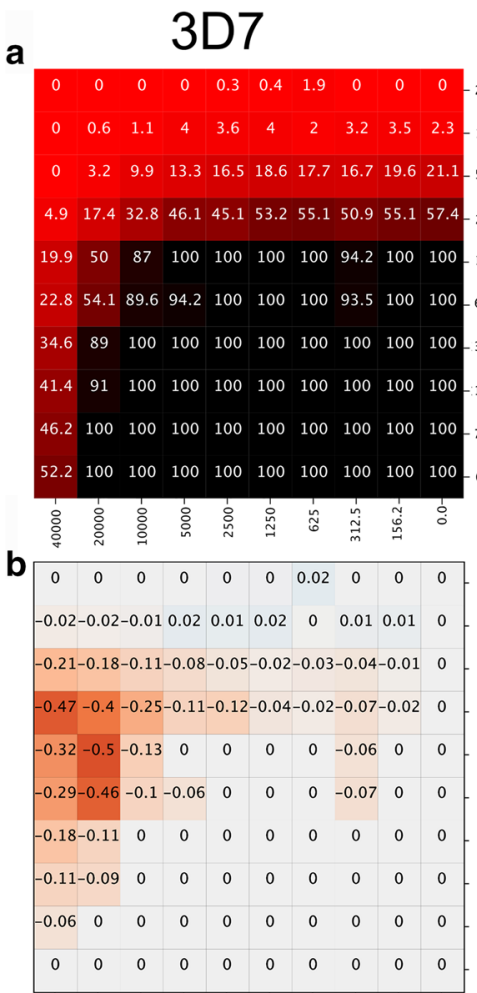

\section{DD2}
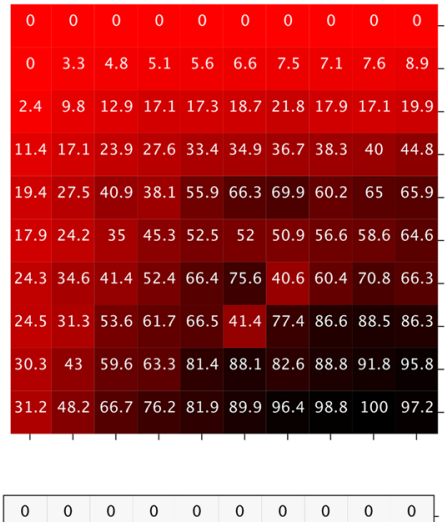

$-0.09-0.06-0.04-0.04-0.03-0.02-0.01-0.02-0.01 \quad 0$ $-0.17-0.1-0.07-0.03-0.03-0.010 .02-0.02-0.03 \quad 0$ $-0.2-0.28-0.21-0.17-0.11-0.1-0.08-0.07-0.05 \quad 0$ $-0.12-0.21-0.25-0.28-0.1 \quad 0 \quad 0.04-0.06-0.01 \quad 0$ $-0.13-0.24-0.3-0.19-0.12-0.13-0.14-0.08-0.06 \quad 0$ $\begin{array}{llll}-0.07-0.14-0.25-0.14 & 0 & 0.09-0.26-0.060 .04 & 0\end{array}$ $-0.07-0.17-0.13-0.14-0.15-0.45-0.09 \quad 0 \quad 0.02 \quad 0$ $-0.01-0.05-0.07-0.13-0.01-0.02-0.13-0.07-0.04 \quad 0$ $\begin{array}{llllllllll}0 & 0 & 0 & 0 & 0 & 0 & 0 & 0.02 & 0.03 & 0\end{array}$

\section{HB3}

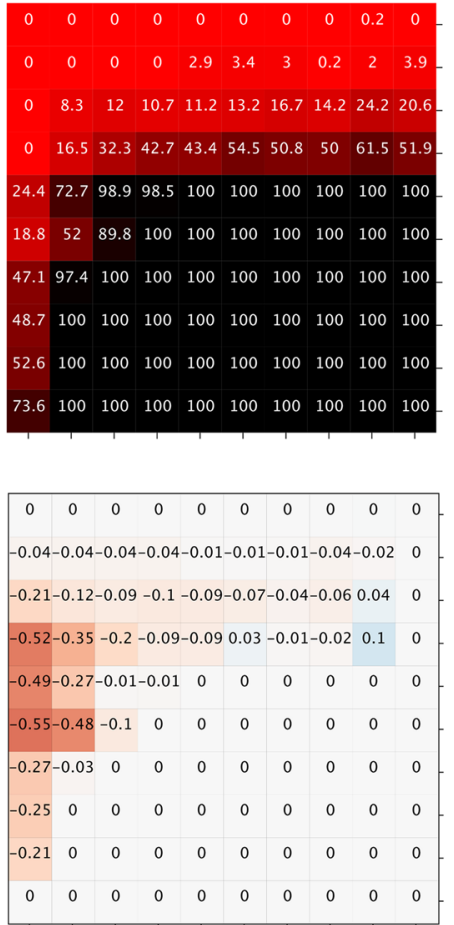

Hydroxyzine
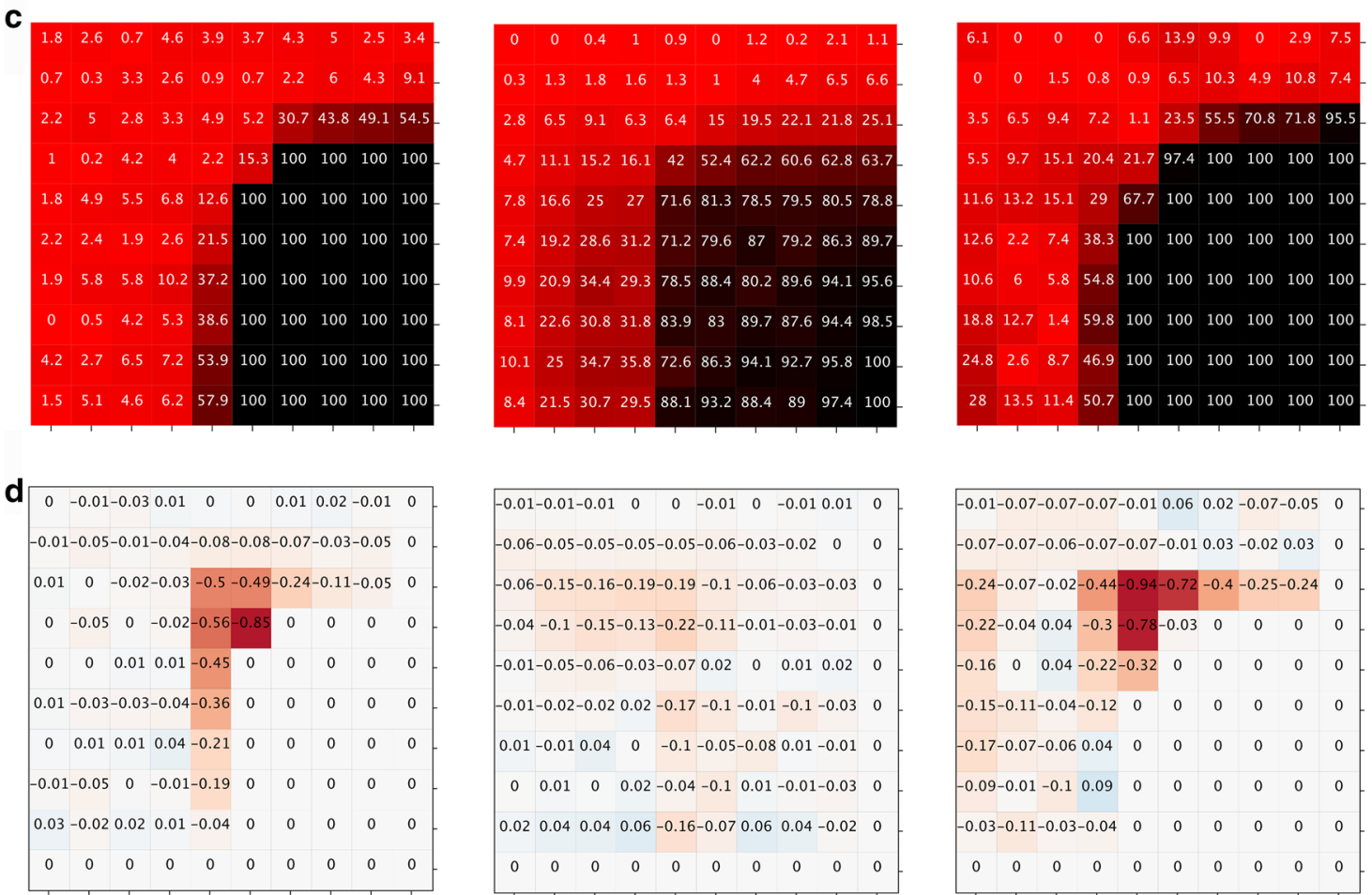

$-0.01-0.07-0.07-0.07-0.010 .06 \quad 0.02-0.07-0.05 \quad 0$ -0.07-0.07-0.06-0.07-0.07-0.01 $0.03-0.020 .03 \quad 0$ $-0.24-0.07-0.02-0.44-0.94-0.72-0.4-0.25-0.24 \quad 0$ $\begin{array}{llllllll}-0.22-0.04 & 0.04 & -0.3-0.78-0.03 & 0 & 0 & 0 & 0\end{array}$ $\begin{array}{llllllll}-0.16 & 0 & 0.04-0.22-0.32 & 0 & 0 & 0 & 0 & 0\end{array}$ $-0.15-0.11-0.04-0.12 \quad 0 \quad 0000000$

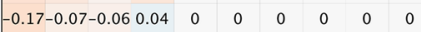

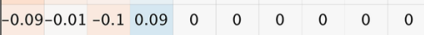

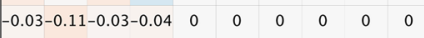
$0 \begin{array}{llllllllll}0 & 0 & 0 & 0 & 0 & 0 & 0 & 0 & 0 & 0\end{array}$

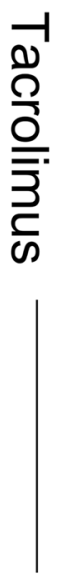



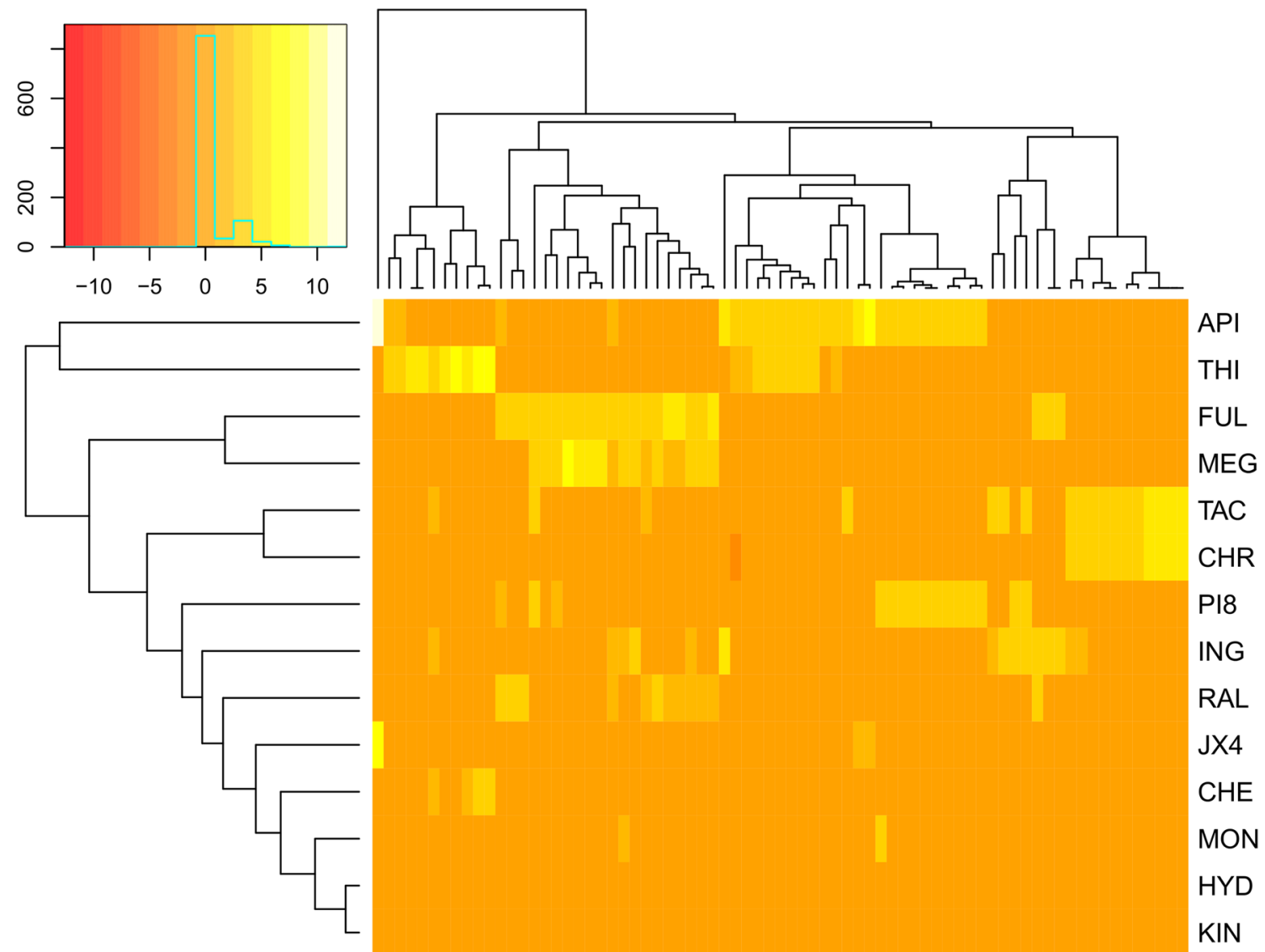

Fig. 5 Pathway annotation of selected agents in the P. falciparum screen. Y axis pathway IDs are explained in Additional file 4: Table S1. Compounds are clustered based on their similarity in pathway bioactivity space, with yellow indicating high enrichment Z-score of a particular pathway, and red indicating lower enrichments

the cut-off chosen is appropriate, given the observed synergy even for established combination therapies.

To address the effect of gamma choice on the validation step, various gamma cut-offs were used and the accuracy of the algorithm based on each of the cut-offs were validated. Table 4 shows the precision and recall based on each of the cut-offs. When gamma cut-offs of 0.975 and 0.995 are used, the precision of the algorithm is 1.98 and 2.02 times better than the training set while recall has been kept as high as 75.5 and $65.1 \%$. It is notable that the training data is selected in a biased way rather than randomly, as it includes current combination therapies and investigational drugs. In case of 0.95 cut-off, the average recall is kept still as high as $62.7 \%$ in average. The precision on the DD2 strain is 1.45 times better than the biased training set. However, in average over the three strains the precision is similar to the training set which was selected with biological insights. Hence the algorithm is almost twice better than biased selection for predicting mild-to-strong or moderate-to-strong synergies. In case of only strong synergy detection the approach is 1.4 times better than biased in the DD2 strain but similar to biased in average for the three strains. To the best of authors' knowledge there is no similar study published elsewhere that has a better precision and recall for predicting synergistic pairs for malaria.

\section{Conclusions}

Recently drug resistance has emerged, and continues to develop, to the existing drug combination therapies for malaria, and hence discovering novel therapies is of much importance [7]. However, testing combinations is costly and time consuming. Systematic approaches towards novel compound combination discoveries can reduce financial cost as it helps to identify compounds in a more informed manner. In this work, 
an integrated transcriptional drug repositioning and machine learning approach was developed to predict synergistic compound combinations for malaria. The transcriptional drug repositioning approach led to the identification of active single agents against malaria. Chromomycin-a3 $\quad($ AC50 $=0.011 \mu \mathrm{M})$, fulvestrant $(0.0667 \mu \mathrm{M})$, apicidin $(0.078 \mu \mathrm{M})$, ingenol $(1.4408 \mu \mathrm{M})$ and tacrolimus $(2.4383 \mu \mathrm{M})$ exhibited full inhibition in low concentrations. The machine learning approach utilized here was trained on a dataset of experimentally assayed compound combinations (including synergistic, antagonistic and additive responses), across three strains of malaria to suggest new compound combinations.

The predicted synergistic compound combinations were experimentally validated, and synergistic compound combinations were identified with overall precision and recall of 83.5 and $65.1 \%$ for mild-to-strong, 48.8 and $75.5 \%$ for moderate-strong and 12.0 and $62.7 \%$ for strong synergies. In all of the predictions recall has been kept high between 50 and $80 \%$ depending the expected synergy level and the strain. Highest precision is achieved for mild-to-strong synergies ranging from 61.3 to $72 \%$. However, as the synergy level expectation is increased to moderate-to-strong or only strong synergies the precision drops to $30-59 \%$ and $4.8-22.7 \%$. Strong novel synergistic compound combinations including combination of tacrolimus with hydroxyzine (average $(\gamma)=0.928$ ) as well as raloxifene and thioridazine (average $(\gamma)=0.932)$ were identified. Moreover, according to the data, targeting independent pathways was more privileged that targeting the same pathway with both compounds in a combination. For retrospective validation, it is notable that tacrolimus and apicidin that were predicted for $P$. falciparum strains have previously been studied for malaria. In vivo validation of apicidin against $P$. berghei malaria in mice is evident from the literature [51]. This retrospective validation provides further assurance of the computational approach presented in this study.
For a single agent to be translated to actual cure, there are several requirements. One of these is that the dosage that activity is occurring, should be lower than maximal safe plasma concentrations of the drugs. The maximal plasma concentration of the single agents are provided in Table 5. In this study, the highest active single agents were apicidin, fulvestrant and chromomycin-a3. The only single agent that is active in lower doses than the maximal safe plasma concentration is apicidin. The AC50 value of apicidin is 74.9, 84.1 and $74.9 \mathrm{nM}$ in 3D7, DD2 and HB3 $P$. falciparum strains while its maximal safe plasma concentration in human is $547.6 \pm 136.6 \mathrm{nM}$. Apicidin at the dose of $500 \mathrm{nM}$ kills in average $97 \%$ of the parasite while it is a safe dose for human. However, apicidin is not approved drug and is not in clinical trials. Hence, entering clinic requires further safety and efficacy studies of apicidin.

The most synergistic compound combinations were tacrolimus-hydroxyzine, tacrolimus-fulvestrant, ingenoltacrolimus, fulvestrant-megestrol and raloxifene-thioridazine. All of the synergies were occurring at doses above the maximal safe plasma concentrations, which renders the current study more of a proof-of-principle than a method to select therapeutically relevant compound combinations directly. A clear definition of required pharmacokinetics and pharmacodynamics properties will also help with the design of drug regimens for dosing anti-malarial drug combinations [52]. Hence, the synergistic pairs will require further investigations in in vivo models to fully evaluate the possibility of transferring them into clinic. Moreover, the generated dataset can be used as a training set for further compound combination predictions. Moreover, the approach is interesting to be followed up with larger synergy prediction candidates (with a different test set).

Accuracy of the predictions and identification of novel single agents and combinations suggests that the integration of machine learning methods with gene

Table 5 Maximum plasma concentration of experimentally validated single agents with activity against malaria

\begin{tabular}{lllll}
\hline Compound & $\begin{array}{l}\text { Maximum plasma concentration, } \\
\mathbf{C}_{\text {Max }}(\mathbf{n g} / \mathbf{m L})\end{array}$ & Molar mass $(\mathbf{g} / \mathbf{m o l})$ & Maximal dose $(\mathbf{n M})$ & Refs \\
\hline FUL & 12.6 & 606.772 & 20.8 & {$[53]$} \\
RAL & 1 & 473.584 & 2.1 & {$[54]$} \\
THI & 205.5 & 370.577 & 1071 & {$[55]$} \\
MEG & 412 & 384.516 & 19.6 & {$[56]$} \\
TAC & 15.8 & 804.018 & 85.3 & {$[57]$} \\
HYD & 32 & 374.904 & $547.6 \pm 136.6$ \\
API & $341.6 \pm 85.2$ & 623.79 & 1.5 & {$[58]$} \\
CHR & 1.85 & 1183.25 & {$[59]$} \\
\hline
\end{tabular}


expression data analysis can lead to a robust platform for predicting novel synergistic compound combinations, in this case applied to malaria.

\section{Additional files}

Additional file 1: Table S2. Predicted synergistic compound combinations for malaria. The predicted probability of being synergistic for each compound pair in each strain.

Additional file 2: Figure S1. Definition of curve classes. Classes of activity [61] were assigned based on growth inhibition curves where -1.1 shows complete curve as well as high efficacy, -1.2 represents complete curve but partial efficacy, -2.1 symbolizes partial curve but high efficacy, - 2.2 represents partial curve and partial efficacy and -2.3 represents partial curve, high efficacy but poor curve fit.

Additional file 3: Figure S2. Pathway enrichment for selected predicted compounds. a) The Figure represents the amount of enrichment (Z-score) of each pathway. The $Y$ axis represents the pathway indices of pathways listed in Additional file 4: Table S1.

Additional file 4: Table S1. Pathway Z-scores for predicted compounds. The table lists pathways from Biosystems that were significantly enriched at least for one of the predicted compounds. The values are Z-scores calculated for each compound compared to background compounds in the training and test set. The Index ID and Biosystems pathway ID is also provided.

\section{Abbreviations}

ACT: artemisinin-based combination therapy; CHR: chromomycin-a3; FUL: fulvestrant; API: apicidin; ING: ingenol; TAC: tacrolimus; JX4: JX-401; RAL: raloxifene; HYD: hydroxyzine; THI: thioridazine; KIN: KIN001-244; PI8: PI 828; MEG: megestrol; MON: monorden; CHE: chelidonine; NCATS: National Center for Advancing Translational Sciences, National Institutes of Health; GEO: Gene Expression Omnibus; GSEA: Gene Set Enrichment Analysis; QC: quality control; TP: true positives; FP: false positives; FN: false negatives; qHTS: quantitative high-throughput screening.

\section{Authors' contributions}

YKM has designed and performed the computational predictions and written the manuscript. RTE has performed the experimental validations. RG supervised experimental validations. $A B$ has supervised the computational work. $A B$ and RG provided feedback for the manuscript. All authors read and approved the final manuscript.

\section{Acknowledgements}

YK and AB thank the European Research Council (ERC Starting Grant 2013 to $A B)$ for funding.

\section{Competing interests}

The authors declare that they have no competing interests.

\section{Availability of data and materials}

All the experimental validation data is publicly available via this link: https:// tripod.nih.gov/matrix-client/?p=1261.

\section{Ethics approval and consent to participate}

Not applicable.

\section{Funding}

This work was supported by European Research Council Grant (ERC Starting Grant 2013 to $A B)$.

\section{Publisher's Note}

Springer Nature remains neutral with regard to jurisdictional claims in published maps and institutional affiliations.
Received: 29 June 2017 Accepted: 24 March 2018

Published online: 11 April 2018

\section{References}

1. World Health Organization. World malaria report. Geneva: World Health Organization; 2015

2. White NJ, Olliaro PL. Strategies for the prevention of antimalarial drug resistance: rationale for combination chemotherapy for malaria. Parasitol Today. 1996;12:399-401.

3. Summers RL, Dave A, Dolstra TJ, Bellanca S, Marchetti RV, Nash MN, et al. Diverse mutational pathways converge on saturable chloroquine transport via the malaria parasite's chloroquine resistance transporter. Proc Natl Acad Sci USA. 2014;111:E1759-67.

4. Nzila AM, Mberu EK, Sulo J, Dayo H, Winstanley PA, Sibley CH, et al. Towards an understanding of the mechanism of pyrimethamine-sulfadoxine resistance in Plasmodium falciparum: genotyping of dihydrofolate reductase and dihydropteroate synthase of Kenyan parasites. Antimicrob Agents Chemother. 2000;44:991-6.

5. World Health Organization. Guidelines for the treatment of malaria. Geneva: World Health Organization; 2015.

6. Mott BT, Eastman RT, Guha R, Sherlach KS, Siriwardana A, Shinn P, et al. High-throughput matrix screening identifies synergistic and antagonistic antimalarial drug combinations. Sci Rep. 2015;5:13891.

7. White N. Antimalarial drug resistance and combination chemotherapy. Philos Trans R Soc Lond B Biol Sci. 1999;354:739-49.

8. Bulusu KC, Guha R, Mason DJ, Lewis RPI, Muratov E, Motamedi YK, et al. Modelling of compound combination effects and applications to efficacy and toxicity: state-of-the-art, challenges and perspectives. Drug Discov Today. 2015;21:225-38.

9. Menden M, Wang D, Guan Y, Mason M, Szalai B, Bulusu K, Yu T, Kang J, Ghazoui Z, Ahsen M, Vogel R, Neto E, Norman T, Tang E, Garnett M, Veroli G, Fawell S, Stolovitzky G, Guinney J, Dry J, Saez-Rodriguez J. Community assessment of cancer drug combination screens identifies strategies for synergy prediction. bioRxiv. 2018;200451. https://doi.org/10.1101/20045 1.

10. Singh N, Misra K. Computational screening of molecular targets in Plasmodium for novel non resistant anti-malarial drugs. Bioinformation. 2009:3:255-62.

11. Swain SS, Sahu MC, Padhy RN. In silico attempt for adduct agent(s) against malaria: combination of chloroquine with alkaloids of Adhatodo vasica. Comput Methods Programs Biomed. 2015;122:16-25.

12. Dasgupta T, Chitnumsub P, Kamchonwongpaisan S, Maneeruttanarungroj C, Nichols SE, Lyons TM, et al. Exploiting structural analysis, in silico screening, and serendipity to identify novel inhibitors of drug-resistant falciparum malaria. ACS Chem Biol. 2009;4:29-40.

13. Lewis R, Guha R, Korcsmaros T, Bender A. Synergy maps: exploring compound combinations using network-based visualization. J Cheminform. 2015;7:1-11.

14. Díaz G, González FA, Romero E. A semi-automatic method for quantification and classification of erythrocytes infected with malaria parasites in microscopic images. J Biomed Inform. 2009:42:296-307.

15. Kinnings SL, Liu N, Tonge PJ, Jackson RM, Xie L, Bourne PE. A machine learning-based method to improve docking scoring functions and its application to drug repurposing. J Chem Inf Model. 2011;51:408-19.

16. Sudheer C, Sohani SK, Kumar D, Malik A, Chahar BR, Nema AK, et al. A support vector machine-firefly algorithm based forecasting model to determine malaria transmission. Neurocomputing. 2014;129:279-88.

17. Iorio F, Rittman T, Ge H, Menden M, Saez-Rodriguez J. Transcriptional data: a new gateway to drug repositioning? Drug Discov Today. 2012;18:350-7.

18. Iskar M, Zeller G, Blattmann P, Campillos M, Kuhn M, Kaminska KH, et al. Characterization of drug-induced transcriptional modules: towards drug repositioning and functional understanding. Mol Syst Biol. 2014;9:662.

19. Liu Y, Zhao H. Predicting synergistic effects between compounds through their structural similarity and effects on transcriptomes. Bioinformatics. 2016;24:1-8.

20. Law GL, Tisoncik-Go J, Korth MJ, Katze MG. Drug repurposing: a better approach for infectious disease drug discovery? Curr Opin Immunol. 2013;25:588-92. 
21. Josset L, Textoris J, Loriod B, Ferraris O, Moules V, Lina B, et al. Gene expression signature-based screening identifies new broadly effective influenza a antivirals. PLOS ONE. 2010;5:e13169.

22. Sirota M, Dudley JT, Kim J, Chiang AP, Morgan AA, Sweet-Cordero A, et al. Discovery and preclinical validation of drug indications using compendia of public gene expression data. Sci Transl Med. 2011;77:96ra77.

23. Library of integrated cellular signatures (LINCS). 2016. http://www.lincs cloud.org/. Accessed 1 Feb 2015.

24. Koutsoukas A, Lowe R, KalantarMotamedi Y, Mussa HY, John BO. In silico target predictions: defining a benchmarking data set and comparison of performance of the multiclass naïve Bayes and Parzen-Rosenblatt. J Cheminform. 2013;53:1957-66.

25. Koutsoukas A, Simms B, Kirchmair J, Bond PJ, Whitmore AV, Zimmer S, et al. From in silico target prediction to multi-target drug design: current databases, methods and applications. J Proteomics. 2011;74:2554-74.

26. Cokol M, Chua HN, Tasan M, Mutlu B, Weinstein ZB, Suzuki Y, et al. Systematic exploration of synergistic drug pairs. Mol Syst Biol. 2011;7:544.

27. KalantarMotamedi Y, Peymani M, Baharvand $\mathrm{H}$, Nasr-Esfahani MH, Bender A. Systematic selection of small molecules to promote differentiation of embryonic stem cells and experimental validation for generating cardiomyocytes. Cell Death Discov. 2016;2:16007.

28. Edgar R, Domrachev M, Lash AE. Gene Expression Omnibus: NCBI gene expression and hybridization array data repository. Nucleic Acids Res. 2002:30:207-10.

29. GEO. GEOImporter. 2012. http://genepattern.broadinstitute.org/gp/addTa sk.jsp?name=urn:Isid:broad.mit.edu:cancer.software.genepattern.modul e.analysis:00042:5\&view=1. Accessed 1 July 2015.

30. Reich M, Liefeld T, Gould J, Lerner J, Tamayo P, Mesirov JP. GenePattern 2.0. Nat Genet. 2006;38:500-1

31. Krupka M, Seydel K, Feintuch CM, Yee K, Kim R, Lin C-Y, et al. Mild Plasmodium falciparum malaria following an episode of severe malaria is associated with induction of the interferon pathway in Malawian children. Infect Immun. 2012:80:1150-5.

32. Subramanian A, Tamayo P, Mootha VK, Mukherjee S, Ebert BL. Gene set enrichment analysis : a knowledge-based approach for interpreting genome-wide. Proc Natl Acad Sci USA. 2005;102:15545-50.

33. Gaulton A, Bellis LJ, Bento AP, Chambers J, Davies M, Hersey A, et al. ChEMBL: a large-scale bioactivity database for drug discovery. Nucleic Acids Res. 2012;40(Database issue):D1100-7.

34. Mestres J, Gregori-Puigjané E, Valverde S, Solé RV. The topology of drugtarget interaction networks: implicit dependence on drug properties and target families. Mol BioSyst. 2009;5:1051-7.

35. Geer LY, Marchler-Bauer A, Geer RC, Han L, He J, He S, et al. The NCBI BioSystems database. Nucleic Acids Res. 2010;38(Database issue):D492-6.

36. Kasprzyk A. BioMart: driving a paradigm change in biological data management. Database (Oxford). 2011;2011:bar049.

37. Svetnik V, Liaw A, Tong C, Culberson JC, Sheridan RP, Feuston BP. Random forest: a classification and regression tool for compound classification and QSAR modeling. J Chem Inf Comput Sci. 2003;43:1947-58.

38. MathWorks. treeBagger. 2012. http://www.mathworks.co.uk/help/stats/ treebagger.html;jsessionid=6f00db9be3dac26e04066a9fd4a2. Accessed 1 July 2015.

39. Yuan J, Cheng KC-C, Johnson RL, Huang R, Pattaradilokrat S, Liu A, et al. Chemical genomic profiling for antimalarial therapies, response signatures, and molecular targets. Science. 2011;333:724-9.

40. Smilkstein M, Sriwilaijaroen N, Kelly JX, Wilairat P, Riscoe M. Simple and inexpensive fluorescence-based technique for high-throughput antimalarial drug screening. Antimicrob Agents Chemother. 2004;48:1803-6.

41. Bennett TN, Paguio M, Gligorijevic B, Seudieu C, Kosar AD, Davidson E, et al. Novel, rapid, and inexpensive cell-based quantification of antimalarial drug efficacy. Antimicrob Agents Chemother. 2004;48:1807-10.

42. Mathews Griner LA, Guha R, Shinn P, Young RM, Keller JM, Liu D, et al. High-throughput combinatorial screening identifies drugs that cooperate with ibrutinib to kill activated B-cell-like diffuse large B-cell lymphoma cells. Proc Natl Acad Sci USA. 2014;111:2349-54.

43. Berenbaum MC. What is synergy? Pharmacol Rev. 1989;41:93-141.

44. Maglott D, Ostell J, Pruitt KD, Tatusova T. Entrez gene: gene-centered information at NCBI. Nucleic Acids Res. 2004;33(Database issue):D54-8.

45. Oakley MS, Anantharaman V, Venancio TM, Zheng H, Mahajan B, Majam V, et al. Molecular correlates of experimental cerebral malaria detectable in whole blood. Infect Immun. 2011;79:1244-53.
46. Alag R, Qureshi IA, Bharatham N, Shin J, Lescar J, Yoon HS. NMR and crystallographic structures of the FK506 binding domain of human malarial parasite Plasmodium vivax FKBP35. Protein Sci. 2010;19:1577-86.

47. Bharatham N, Chang MW, Yoon HS. Targeting FK506 binding proteins to fight malarial and bacterial infections: current advances and future perspectives. Curr Med Chem. 2011;18:1874-89.

48. Maluccio M, Sharma V, Lagman M, Vyas S, Yang H, Li B, et al. Tacrolimus enhances transforming growth factor-beta1 expression and promotes tumor progression. Transplantation. 2003;76:597-602.

49. Omer FM, Riley EM. Transforming growth factor beta production is inversely correlated with severity of murine malaria infection. J Exp Med. 1998;188:39-48.

50. Omer FM, de Souza JB, Corran PH, Sultan AA, Riley EM. Activation of transforming growth factor by malaria parasite-derived metalloproteinases and a thrombospondin-like molecule. J Exp Med. 2003;198:1817-27.

51. Darkin-Rattray SJ, Gurnett AM, Myers RW, Dulski PM, Crumley TM, Allocco $J$, et al. Apicidin: a novel antiprotozoal agent that inhibits parasite histone deacetylase. Proc Natl Acad Sci USA. 1996;93:13143-7.

52. Li Q, Hickman MR. Pharmacokinetics and pharmacodynamics of antimalarial drugs used in combination therapy. Sharjah: Bentham Science Publishers; 2015. p. 500.

53. Robertson JFR, Harrison M. Fulvestrant: pharmacokinetics and pharmacology. Br J Cancer. 2004;90(Suppl 1):S7-10.

54. Czock D, Keller F, Heringa M, Rasche FM. Raloxifene pharmacokinetics in males with normal and impaired renal function. Br J Clin Pharmacol. 2005;59:479-82.

55. Ravic M, Warrington S, Boyce M, Dunn K, Johnston A. Repeated dosing with donepezil does not affect the safety, tolerability or pharmacokinetics of single-dose thioridazine in young volunteers. Br J Clin Pharmacol. 2004;58(Suppl 1):34-40.

56. FDA. MEGACE ${ }^{\circledR}$ ES Megestrol acetate $625 \mathrm{mg} / 5 \mathrm{~mL}$ oral suspension.https ://www.accessdata.fda.gov/drugsatfda_docs/label/2007/021778s002 s003lbl.pdf. Accessed 31 Jan 2018.

57. Rogers CC, Alloway RR, Alexander JW, Cardi M, Trofe J, Vinks AA. Pharmacokinetics of mycophenolic acid, tacrolimus and sirolimus after gastric bypass surgery in end-stage renal disease and transplant patients: a pilot study. Clin Transplant. 2008:22:281-91.

58. Public Assessment Report-Scientific discussion Hydroxyzinhydrochlorid EQL Pharma (Hydroxyzine hydrochloride) Film-coated tablet, 25 mg; 2014. http://mri.cts-mrp.eu/download/DK_H_2313_001_PAR.pdf. Accessed 31 Jan 2018.

59. Soo Shin B, Dong Yoo S, Hwan Kim T, Bulitta JB, Landersdorfer CB, Cheol Shin J, et al. Quantitative determination of absorption and first-pass metabolism of apicidin, a potent histone deacetylase inhibitor. DRUG Metab Dispos. 2014;42:974-82.

60. Garattini S. Advances in pharmacology and chemotherapy, Vol. 12. Academic Press; 1975.

61. Wang $Y$, Nguyen T, Huang R, Jadhav A, Southall NT. Enabling the large-scale analysis of quantitative high-throughput screening data. In: Seethala R, Zhang L, editors. Handbook of drug screening. 2nd ed, Chap 19. New York: Taylor \& Francis Group; 2016. pp. 454-76

Ready to submit your research? Choose BMC and benefit from

- fast, convenient online submission

- thorough peer review by experienced researchers in your field

- rapid publication on acceptance

- support for research data, including large and complex data types

- gold Open Access which fosters wider collaboration and increased citations

- maximum visibility for your research: over 100M website views per year

At BMC, research is always in progress.

Learn more biomedcentral.com/submissions 